The Impact of Large-Scale Surveys on Pulsating Star Research

ASP Conference Series, Vol. 203, 2000

L. Szabados \& D. W. Kurtz, eds.

\title{
Understanding Pulsations in OB Stars
}

\author{
L. A. Balona \\ South African Astronomical Observatory, P.O. Box 9, Observatory \\ 7935, Cape Town, South Africa
}

\begin{abstract}
Our understanding of pulsations in $\beta$ Cep, SPB and $\zeta$ Oph stars is discussed. We do not understand why there are no $\beta$ Cep stars earlier than O9, though the models show that the instability strip extends to hotter stars. The main problem among the SPB stars is why rotation has a strong inhibiting effect on pulsation. The $\zeta$ Oph stars are an extension of the $\beta$ Cep and SPB stars to higher spherical harmonic degrees. The pulsational stability of models of B stars to modes of high degree is discussed. Results are compared with two well observed $\zeta \mathrm{Oph}$ stars. Although there is good agreement with $\zeta$ Oph itself, the periods of the high-degree modes in $\alpha$ Vir are incompatible with model predictions.
\end{abstract}

\section{Introduction}

Convection does not play a role in the pulsational stability of upper main sequence stars. These stars are therefore ideally suited to test our understanding of stellar pulsation. Instability in these stars is caused by an opacity bump at about $200000 \mathrm{~K}$ due to a huge number of absorption lines produced by transitions in the iron-group ions. Dziembowski \& Pamyatnykh (1993) were the first to calculate the position of the instability strip corresponding to the $\beta$ Cep stars. Later, Dziembowski, Moskalik, \& Pamyatnykh (1993) showed that instability due to long period $\mathrm{g}$ modes was also present in models of mid-B stars, and calculated the position of the instability strip for SPB variables. The efficiency of the driving mechanism is very sensitive to the abundances of iron-group elements. A detailed study of pulsation in the upper main sequence stars, which includes the effect of different opacities, has been recently presented by Pamyatnykh (1999). At $\mathrm{Z}=0.01$ the $\beta$ Cep instability strip almost vanishes, though the SPB domain is still present. The SPB domain vanishes for $Z<0.006$.

The fact that the models show two regions of instability, one of short period corresponding to the $\beta$ Cep stars and another of long period corresponding to the SPB stars, deserves some explanation. A necessary condition for pulsational instability is that the thermal time scale of the driving zone should be comparable to the period of pulsation. At the same time, the pressure eigenfunction must be flat and large in this zone. This combination is fulfilled in the $\beta$ Cep stars in which the driving zone is near the surface and the thermal time scale relatively short, so that long-period g modes are stable. It is also fulfilled in the deeper driving zones of cooler stars where the time scale is relatively long, leading to instability of long-period g modes corresponding to SPB variables. 
There exists a group of stars which, though not photometrically variable, show line profile variations expected from pulsations in modes of high spherical harmonic degree, $\ell$. These are the $\zeta$ Oph stars (Balona 1989). Balona \& Dziembowski (1999) have recently computed models of pulsations in these stars up to $\ell=24$. In this paper we briefly summarise these results and discuss our current understanding of pulsations among upper main sequence stars.

\section{The $\beta$ Cep and SPB Stars}

A $\beta$ Cep star may be defined as a B-type star with one or more periods which are equal to, or shorter than, the fundamental radial mode. The location of the stars in the HR diagram is within the instability strip calculated using OP opacities for composition $X=0.70, Z=0.02$. The least evolved stars, particularly the $\beta$ Cep stars in NGC 6231 , fall somewhat outside the instability strip if the OPAL opacities are used (Pamyatnykh 1999). From this we can conclude that observations agree with models within the uncertainties in the opacities. The most striking discrepancy between observations and the models lies in the absence of pulsating stars hotter than 09. This is certainly real, since efforts have been made to detect $\beta$ Cep pulsations in a number of $O$ stars, without success. Clearly, there is some essential physics missing from the models of the hottest stars.

It may seem rather surprising that an Hipparcos survey for variability resulted in only 4 new $\beta$ Cep variables, though 72 new SPB stars were discovered (Waelkens et al. 1998). The reason for the low number of new $\beta$ Cep stars detected by Hipparcos is most likely a selection effect. Ground based surveys are more likely to detect stars with periods of hours. On the other hand, detection of small amplitude variables with periods of the order of a day is particularly difficult from the ground owing to the daily aliasing effect. The Hipparcos sampling therefore favours detection of SPB stars.

The SPB, or 53 Per stars, may be defined as stars which we believe are pulsating (e.g. because they show multiperiodicity) and for which the periods are longer than the fundamental radial mode. The newly discovered SPB stars nicely cover the predicted instability strip (Pamyatnykh 1999). The periods, too, are within the range given by the models, though detailed comparisons need to be made. There is, however, a problem in mode selection: why is it that most of the observed SPB stars pulsate in only very few modes (often just one mode), while the models predict many unstable modes in the same period range?

It has been known for a long time that SPB stars are intrinsically slow rotators. This is strikingly confirmed by the Hipparcos survey of SPB stars. Since this is a purely photometric survey, there is clearly no bias in selecting SPB stars that are slow rotators. Yet the distribution of projected rotational velocities, $v \sin i$, among these stars is quite different from a sample of $\mathrm{B}$ dwarfs and giants with the same range of spectral type (Fig. 1). There is no doubt that, for some reason, the pulsational amplitudes are greatly influenced by rotation. There are practically no SPB stars with $v \sin i>250 \mathrm{~km} \mathrm{~s}^{-1}$.

Ushomirsky \& Bildsten (1998) have studied the effect of rotation on g modes of B-type stars. They suggest that rapid rotation distorts the star and changes the thermal time scale in the driving zone so that it no longer matches the 


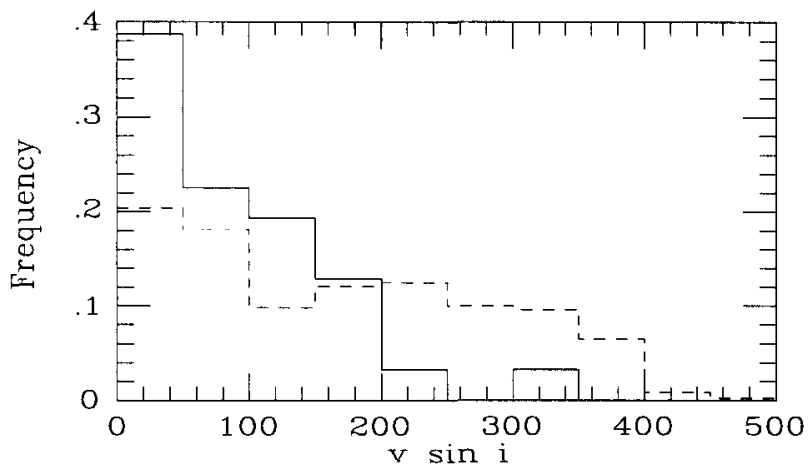

Figure 1. The distribution of projected rotational velocity, $v \sin i$ $\left(\mathrm{km} \mathrm{s}^{-1}\right)$, for 612 normal B3-B9 dwarfs and giants (solid line) and for 31 SPB stars (dashed line). Both distributions are normalised to unit area.

period of pulsation. Therefore rapid rotation tends to stabilise modes which are unstable in a non-rotating star and, conversely, destabilises g modes which are stable in the absence of rotation. Since $g$ modes are strongly confined to the equator in rapidly rotating stars, they are less likely to be detected because the favoured viewing angle is strongly peaked to $i=90^{\circ}$. This is the only explanation currently available for the strong dependence of amplitude on $v \sin i$.

\section{Modes of High Degree}

Until recently, only modes of low degree (typically $\ell<4$ ) have been studied in models of OB stars. Line profile variations of some rapidly rotating B-type stars and $\delta$ Sct stars show travelling subfeatures which could be understood as pulsations of high degree. These moving bumps nearly always travel from blue to red, indicating prograde modes. Why prograde modes of high degree should be more unstable than retrograde modes is an interesting, unsolved, question.

Recently, Balona \& Dziembowski (1999) have studied the stability of stellar models to values of $\ell$ up to $\ell=24$. In non-rotating models of these stars, modes of high order with periods similar to those of low order are also unstable. Thus one may expect the $\beta$ Cep, SPB and $\delta$ Sct stars to show evidence of high degree modes, as indeed are seen in the $\zeta \mathrm{Oph}$ stars and some of the more rapidly rotating $\delta$ Sct stars.

The regions of instability for some modes of high degree are shown in Fig. 2 for models with $Z=0.02$. The extent of the $\beta$ Cep instability strip reaches a maximum at about $\ell=4$ and vanishes at $\ell=16$. On the other hand, the SPB instability strip reaches maximum size at about $\ell=8$ and vanishes only for $\ell>24$. Notice that there is a strip of SPB variability at high temperatures for $\ell=2$, a fact already pointed out by Pamyatnykh (1998). The overlap between the $\beta$ Cep and SPB instability strips increases with $\ell$ such that for $\ell>6$ there is almost complete overlap except for the less luminous stars. 


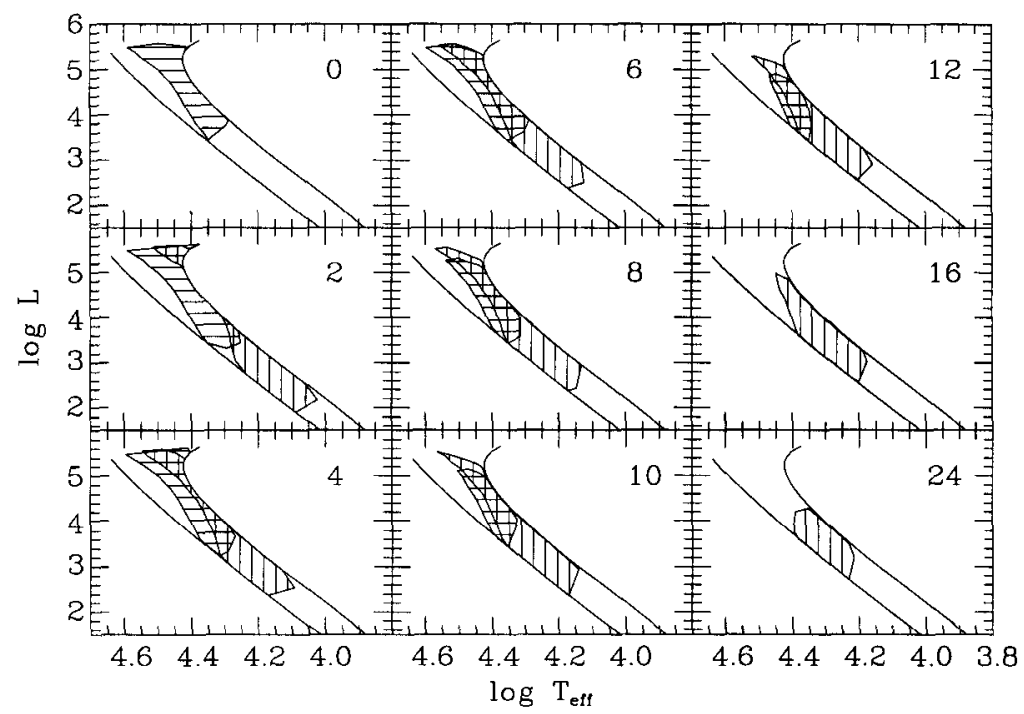

Figure 2. The instability strip for short-period oscillations (horizontally hatched area) and long-period oscillations (vertically hatched area) for spherical harmonic degrees $\ell=0$ to 24 . The ZAMS and the end of core hydrogen burning are shown. The value of $\ell$ is given in each panel.

Observations of high degree modes are rather scarce because a mediumsized telescope is required to obtain the high signal-to-noise data required to detect such modes. In all but two OB stars, the data is merely a detection rather than an actual determination of periods. The best-studied star of this kind is $\zeta$ Oph itself, thanks to a multi-longitude spectroscopic and photometric campaign by Kambe et al. (1997). The other star is $\alpha$ Vir, observed by Smith (1985), but in need of re-analysis using modern techniques.

The biggest problem in trying to compare the models with observations is stellar rotation. The models are calculated for non-rotating stars, but the stars in which modes of high degree are suspected are all rapid rotators. The frequency that is observed, $f$, is related to the frequency in the co-rotating frame, $f_{0}$, by the relationship $f=f_{0}-m \Omega$, where $m$ is the azimuthal spherical harmonic order and $\Omega$ is the frequency of rotation of the star. In order to compare with the frequencies calculated by non-rotating models, we need to calculate $f_{0}$, which means we need to know $m$ and $\Omega$. The value so-determined is affected by Coriolis and centrifugal forces and a further, smaller correction is required.

Balona \& Kambe (1999) analysed the line profile data of Kambe et al. (1997) for $\zeta$ Oph. This star has two oscillations at frequencies of $f_{1}=11.89$ $\mathrm{d}^{-1}$ and $f_{2}=7.19 \mathrm{~d}^{-1}$. Mode identification was performed by phasing the line profiles with each of the two frequencies in turn, treating the other as pure noise. A large grid of line profiles was calculated in which all six pulsational parameters for a given $(\ell, m)$ were varied for best fit. Having established the best-fitting parameters for the individual frequencies, $f_{1}$ and $f_{2}$, a two-frequency 
solution was performed to obtain the optimum parameters. Balona \& Kambe (1999) found that the goodness of fit was rather insensitive to $\ell$ and $m$, and that several equally good solutions were possible for each frequency. The nominally best solution was $\ell=8, m=-8$ for $f_{1}$, and $\ell=4, m=-4$ for $f_{2}$ with a rather high angle of inclination. An estimate of $\Omega$ was obtained from the projected rotational velocity and the radius of the star. The frequencies in the corotating frame were found to be $f_{1,0} \approx 3.9 \mathrm{~d}^{-1}$ and $f_{2,0} \approx 3.2 \mathrm{~d}^{-1}$. Both these values are within the range calculated by non-rotating models of the star. The conclusion is that $\zeta$ Oph can be understood as a $\beta$ Cep star with two modes of high degree.

In some ways, the other well observed star, $\alpha$ Vir, should be easier to interpret because its physical parameters have been accurately determined. It is a double-lined spectroscopic binary, from which we can determine the mass and angle of inclination. It has a large parallax and good interferometric angular diameter, from which the luminosity and temperature can be accurately determined. Smith (1985) detected two modes with $f_{1}=3.68 \mathrm{~d}^{-1}$ with $\ell=-m=8$, and $f_{2}=7.50 \mathrm{~d}^{-1}$ with $\ell=-m=16$. The frequency of rotation is $\Omega=0.43$ $\mathrm{d}^{-1}$. The frequencies in the co-rotating frame are therefore $f_{1,0}=0.24 \mathrm{~d}^{-1}$ and $f_{2,0}=0.62 \mathrm{~d}^{-1}$. These frequencies are at least a factor of two smaller than the unstable modes predicted for the appropriate effective temperature and luminosity. Clearly, there is a rather serious problem which deserves further attention. Perhaps the weakest point is mode identification which could easily resolve the problem. It would be important to re-observe the star or re-analyse Smith's data with modern techniques.

\section{Conclusions}

Our good understanding of pulsations in OB stars can, in many ways, be attributed to the lack of convection in the outer layers of these stars. The periods and location of these stars in the HR diagram are in very good agreement with the models. Extension of the calculations to modes of high degree also explain the $\zeta$ Oph stars. The main difficulty in comparing the periods of $\zeta$ Oph stars with periods from the models is the lack of mode identification. The number of azimuthal modes increases as $2 \ell+1$. The precise value of $m$ is required for a direct comparison with the period predicted from models. Unfortunately, it does not seem to be possible to determine $\ell$ and $m$ with any degree of accuracy when $\ell$ is large, even in the best observed stars. This may be a fundamental limitation.

Apart from the general, unsolved, problem of mode selection, there are two outstanding problems in OB star pulsations: why stars earlier than O9 do not pulsate in modes of low degree, and the effect of rotation on the stability of long period g modes in SPB stars. In both cases there is no doubt that some essential physics is missing in the models. Another interesting problem is why only prograde modes are seen in the $\zeta$ Oph stars. From the observational point of view, there is a need for observing more $\zeta$ Oph stars in order to probe the extent of the instability strip. The aim of such observations should not merely be a detection of these modes, as is usually done, but should include a proper determination of the periods if they are to be truly useful. 


\section{References}

Aerts, C., Balona, L. A., \& Waelkens, C. 1989, in ASP Conf. Ser. Vol. 11, Confrontation between Stellar Pulsation and Evolution, ed. C. Cacciari \& G. Clementini (San Francisco: ASP), 290

Balona, L. A. 1989, in ASP Conf. Ser. Vol. 11, Confrontation between Stellar Pulsation and Evolution, ed. C. Cacciari \& G. Clementini (San Francisco: ASP), 245

Balona, L. A. \& Dziembowski, W. A. 1999, MNRAS, in press

Balona, L. A. \& Kambe, E. 1999, MNRAS, in press

De Mey, K., Aerts, C., Waelkens, C., Cranmer, S. R., Schrijvers, C., Telting, J. H., Daems, K., \& Meeus, G. 1997, A\&A, 324, 1096

Dziembowski, W. A. \& Pamyatnykh, A. A. 1993, MNRAS, 262, 204

Dziembowski, W. A., Moskalik, P., \& Pamyatnykh, A. A. 1993, MNRAS, 265, 588

Kambe, E., Hirata, R., Ando, H., Cuypers, J., Kato, H. M., Kennelly, E. J., Walker, G. A. H., Stefl, S., \& Tarasov, A. E. 1997, ApJ, 481, 406

Pamyatnykh, A. A. 1998, in ASP Conf. Ser. Vol. 135, A Half-century of Stellar Pulsation Interpretations, ed. P. A. Bradley \& J. A. Guzik (San Francisco: ASP), 268

Pamyatnykh, A. A. 1999, Acta Astron., 49, 119

Smith, M. A. 1985, ApJ, 297, 206

Ushomirsky, G. \& Bildsten, L. 1998, ApJ, 497, L101

Waelkens, C., Aerts, C., Kestens, E., Grenon, M., \& Eyer, L. 1998, A\&A, 330, 215

\section{Discussion}

Marten van Kerkwijk: 1. You mention that SPB stars seem to rotate slowly. Could this, in part, be a selection effect, since Be stars are not included?

2. For the inclination of $\alpha \mathrm{Vir}$, did you use the orbital inclination? Is there evidence for synchronous rotation which might support this?

Luis Balona: 1. There are, in fact, very few periodic mid- to late-Be stars, so the inclusion of these as SPB stars would not remove the discrepancy in the distribution of projected rotational velocities. There are distinct differences between the light curves of periodic Be stars ( $\lambda$ Eri stars) and SPB stars, among the most obvious being the presence of double-wave light curves and large amplitude fluctuations. It is most unlikely that the same mechanism is in operation in both types of star, so they must be considered as distinct classes.

2. The axial inclination of the primary in $\alpha$ Vir is assumed to be the same as the orbital inclination. There are, indeed, reasons to believe that supersynchronicity is present in the orbital and rotational periods (Smith 1985), making this assumption more plausible.

Conny Aerts: We have found $\ell$ as high as 6 from preliminary mode identification of SPB stars using Geneva photometry. Therefore there is no strict division 
between SPB and $\zeta$ Oph stars at $\ell=2$ or even $\ell=4$. Also, $\beta$ Cep stars with moving bumps do exist ( $\kappa$ Sco, $\lambda$ Sco, $\beta$ Cen, $\epsilon$ Per) which can be considered as intermediate objects.

Luis Balona: It is certainly interesting that a degree as high as $\ell=6$ can still be detected photometrically. The division between $\beta$ Cep stars and $\zeta$ Oph stars, or SPB stars and $\zeta$ Oph stars, is purely arbitrary, though it is a quite convenient observational description. In future, perhaps one should drop the term $\zeta \mathrm{Oph}$ and simply refer to $\beta$ Cep stars or SPB stars with modes of high degree.

Oliveira Kepler: The lack of observed $\beta$ Cep stars among the O-type stars may be due to loss of reflection at the outer boundary condition due to radiative losses. Perhaps the same effect could account for the lack of SPB stars with high rotational velocities.

Luis Balona: The solution you suggest may certainly be the correct one for the lack of hot $\beta$ Cep stars, though I am not aware of its application to these stars. I would be surprised if the lack of rapid rotators among SPB stars can be attributed to the same cause.

Yanqin Wu: In the stability analysis, modes of low as well as high degree are unstable. Do you have any idea why in $\zeta$ Oph stars only modes of high degree are observed?

Luis Balona: It is true that in $\zeta \mathrm{Oph}$ and $\alpha \mathrm{Vir}$, only modes of high degree are presently visible, but as already mentioned, modes of both low and high degree are present in a few $\beta$ Cep stars such as $\lambda$ Sco (De Mey et al. 1997) and $\kappa$ Sco (Aerts, Balona, \& Waelkens 1989). We do not yet understand the problem of mode selection, so we cannot explain why certain modes are excited and others are not. 